\title{
Precise measurement of $K$-shell fluorescence yield in iridium: An improved test of internal-conversion theory
}

\author{
N. Nica, ${ }^{*}$ J. C. Hardy, V. E. Iacob, and J. R. Montague ${ }^{\dagger}$ \\ Cyclotron Institute, Texas A\&M University, College Station, Texas 77843, USA \\ M. B. Trzhaskovskaya \\ Petersburg Nuclear Physics Institute, Gatchina RU-188300, Russia \\ (Received 27 January 2005; published 27 May 2005)
}

\begin{abstract}
We have measured the total intensity of $K \mathrm{x}$ rays relative to $129.4-\mathrm{keV} \gamma$ rays from decay of the second excited state in ${ }^{191}$ Ir. This $(M 1+E 2)$ transition was observed following the $\beta$ decay of $15.4-\mathrm{d}{ }^{191}$ Os. Our measured ratio yields the result $\alpha_{K} \omega_{K}=2.044(11)$. When combined with a recent measurement of the same ratio for the 80.2-keV M4 transition from ${ }^{193} \mathrm{Ir}^{m}$, this result strongly confirms the need for the $K$-shell hole to be included in calculations of internal-conversion coefficients $\alpha_{K}$. Since the $\alpha_{K}$ value calculated for the ${ }^{191} \operatorname{Ir}$ transition is virtually independent of the hole treatment, our result also yields a model-independent value for the iridium fluorescence yield, $\omega_{K}=0.954(9)$.
\end{abstract}

DOI: 10.1103/PhysRevC.71.054320

PACS number(s): $23.20 . \mathrm{Nx}, 27.80 .+\mathrm{w}$

\section{INTRODUCTION}

In a recent paper [1], we reported a precise determination of $\alpha_{K}$, the $K$-shell internal-conversion coefficient (ICC), for the $80.2-\mathrm{keV} M 4$ transition from ${ }^{193} \mathrm{Ir}^{m}$. That result clearly demonstrated for the first time that an important requirement for the calculation of ICCs is that the final-state electron wave function must be computed in a field that includes the vacancy in the atomic subshell from which the conversion electron was emitted.

Calculations of tabulated ICCs over the years [2-6] have not always incorporated that vacancy, and indeed Band et al., the authors of the most recent tables [6], specifically chose to omit the hole in their calculations, basing their decision on an earlier comparison with experimental data [7], which indicated that better overall agreement with experimental ICCs would result. A subsequent survey of ICC data [8], which compared experimental results to the values tabulated by Band et al. and also to values similarly calculated but with the hole included, found that the former agreed to within a few tenths of a percent, while the latter disagreed by about $1 \%$. However, the body of world ICC data included very few measurements of high precision $(\leqslant 1 \%)$, and it was only in the average over many different transitions that a discrepancy between experiment and theory could be discerned at all. This was hardly definitive evidence for one calculation over the other.

The measured 80.2-keV M4 transition from ${ }^{193} \mathrm{Ir}^{m}$ provided that definitive evidence [1]. Since the transition energy is very close to the $76.1-\mathrm{keV} K$-shell binding energy in iridium, calculated values for the $K$-shell ICC, $\alpha_{K}$, differ from one another by more than $10 \%$ depending on whether the hole is incorporated in the calculation or not. Our published result for

\footnotetext{
*Email address: nica@comp.tamu.edu

${ }^{\dagger}$ REU summer student from Colby College, Waterville, Maine 04901 .

${ }^{\ddagger}$ URL:http://cycnt.tamu.edu/
}

$\alpha_{K}$, quoted to a precision of $0.8 \%$, agreed completely with the calculation that includes the hole, and ruled out the no-hole value by more than ten standard deviations.

One small potential loophole remained though. In the ${ }^{193} \mathrm{Ir}^{m}$ experiment, we compared the total number of $K \mathrm{x}$ rays, $N_{K}$, observed from the $80.2-\mathrm{keV}$ transition to the total number of $\gamma$ rays, $N_{\gamma}$. This ratio relates to $\alpha_{K}$ via the simple relationship

$$
\alpha_{K} \omega_{K}=\frac{N_{K}}{N_{\gamma}} \frac{\epsilon_{\gamma}}{\epsilon_{K}}
$$

where $\epsilon_{\gamma}$ and $\epsilon_{K}$ are the detector efficiencies for the $\gamma$ rays and $\mathrm{x}$ rays, respectively, and $\omega_{K}$ is the $K$-shell fluorescence yield for iridium. Unfortunately, the $\omega_{K}$ for iridium has never been directly measured, so in order to extract $\alpha_{K}$ from this equation, we were forced to take the fluorescence yield from tables [9] based on a semi-empirical fit (as a function of $Z$ ) to existing data, which are actually quite sparse for $Z>63$ (see Ref. [10]). The tabulated value, $\omega_{K}=0.958(4)$, was quoted with sufficient precision that we could comfortably use it to extract a $0.8 \%$ result for $\alpha_{K}$, but we considered that our conclusions would become even more secure if we could make a direct measurement of the iridium fluorescence yield and use that value instead. This paper presents just such a measurement.

Here we use the same approach we used for the ${ }^{193} \operatorname{Tr}^{m}$ decay, measuring the intensity ratio of $K \mathrm{x}$ rays to $\gamma$ rays, but we now focus on a $129.4-\mathrm{keV}(M 1+E 2)$ transition in ${ }^{191} \mathrm{Ir}$. Since the calculated value of $\alpha_{K}$ for this transition is virtually independent of the treatment of the $K$-shell vacancy, Eq. (1) can in this case be used to extract an experimental result for $\omega_{K}$. Alternatively, the ratio of the products $\alpha_{K} \omega_{K}$ from the ${ }^{191} \mathrm{Ir}$ and ${ }^{193} \mathrm{Ir}^{m}$ experiments can be used to obtain the ratio of ICCs entirely independently of the fluorescence yield, which cancels in the ratio. This experimental ICC ratio can then be compared with ICC calculations that use different treatments for the $K$-shell vacancy. 


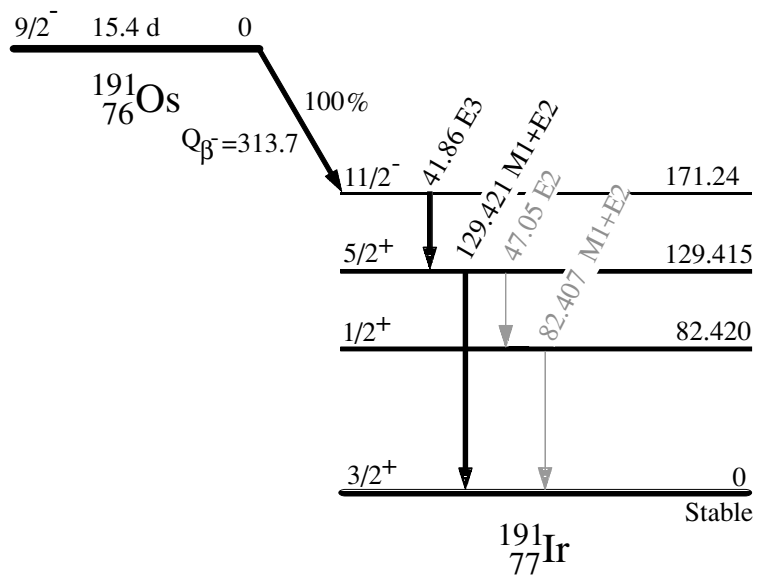

FIG. 1. Decay scheme for the $\beta$ decay of ${ }^{191}$ Os, including the $\gamma$ transitions that occur subsequently in its daughter, ${ }^{191}$ Ir. The information is taken from Ref. [11]. Note that the strong transitions are shown with heavy arrows. The weak $47-\mathrm{keV}$ transition accounts for less than $0.4 \%$ of decay from the $129-\mathrm{keV}$ state (including the effects of internal conversion).

This paper is closely related to our previous one [1], so we only deal extensively here with aspects of the measurement that are significantly different. However, because this is an independent measurement, we still pay attention to those details that affect the subpercent precision we quote. Sections II and III describe the experiment and data analysis, respectively. Section IV presents the results, and we conclude with some discussion of the impact of our results in Sec. V.

\section{EXPERIMENT}

We used the same experimental method and setup as in our previous measurement, described in Ref. [1]. The 15.4-day $\beta$ decaying isotope, ${ }^{191} \mathrm{Os}$, was produced by neutron capture, and its decay was observed with a HPGe detector of precisely calibrated efficiency. Figure 1 shows the unique $\beta$-decay branch from ${ }^{191} \mathrm{Os}$, and the subsequent $\gamma$ transitions in its daughter ${ }^{191}$ Ir. Only two of the $\gamma$ transitions in ${ }^{191}$ Ir actually play a significant role, those with energies of 41.8 and $129.4 \mathrm{keV}$. The strength of the 47.1-keV transition (including its internalconversion component) accounts for less than $0.4 \%$ of the total decay of the 129.4-keV level [11], so both it and the 82.4-keV transition that follows it contribute little to the observed spectrum. Furthermore, both the (strong) $41.8-\mathrm{keV}$ transition and the (weak) 47.1-keV one are almost $100 \%$ converted, but entirely in the $L$ shell and beyond; only the (strong) $129.4-\mathrm{keV}$ and (weak) 82.4-keV transitions are converted in the $K$ shell. Thus, the $K \mathrm{x}$ rays observed in this decay are dominated by those from internal conversion of the 129.4-keV transition, and with a small correction, they can be used in Eq. (1) together with the 129.4-keV $\gamma$ rays to determine the value of $\alpha_{K} \omega_{K}$ for this transition.

However, for this result to be useful in comparison with calculated $\alpha_{K}$ values, it is essential to know the mixing ratio between the E2 and $M 1$ components in the 129.4$\mathrm{keV}$ transition. The mixing ratio has been determined on
TABLE I. Sources prepared by neutron activation, their type, mass, and the calculated self-absorption for $K_{\alpha}$ and $K_{\beta}$ x rays, and the $129.4-\mathrm{keV} \gamma$ ray.

\begin{tabular}{lccccc}
\hline \hline Source & Type & \multirow{2}{*}{$\begin{array}{c}\text { Mass } \\
(\mathrm{mg})\end{array}$} & \multicolumn{3}{c}{ Self-absorption $(\%)$} \\
\cline { 4 - 6 } & & & $K_{\alpha}$ & $K_{\beta}$ & $\gamma$ \\
\hline & & & & & \\
& & & & & \\
$O 1$ & Osmium chloride & 0.48 & 0.05 & 0.06 & 0.04 \\
$O 2$ & Osmium oxide & 0.60 & 0.37 & 0.47 & 0.29 \\
\hline \hline
\end{tabular}

a number of occasions, but some of these determinations themselves depended on calculated conversion coefficients. This important issue is discussed in Sec. IV A. In what immediately follows, we describe our source preparation and data acquisition, and then we deal with impurities and small corrections.

\section{A. Source preparation}

We prepared sources of natural osmium from powders of osmium oxide $\mathrm{OsO}_{2}$ and osmium chloride hydrate $\mathrm{OsCl}_{3} \times$ $3 \mathrm{H}_{2} \mathrm{O}$ purchased from ESPICorp Inc. The granularity of the powders was less than $40 \mu \mathrm{m}$, while the purity was 99.9\% relative to the other rare earths only, but not including contamination from reducing agents or crucible materials. The chloride is very soluble in water, and we evaporated a drop of solution on a $2.5-\mathrm{cm}$ square of $75-\mu \mathrm{m}$-thick mylar film, on which we had previously dried insulin in order to ensure a uniform thin chloride layer. Because the oxide is not soluble, we milled it to thinner powder of approximately micron size and sprinkled it uniformly on the mylar backing. We checked the size and uniformity of the deposited material with a microscope. The samples were then sealed with very thin plastic adhesive tape.

A number of such samples were placed in a thermal-neutron flux of $\sim 7 \times 10^{12} \mathrm{n} / \mathrm{cm}^{2} \mathrm{~s}$ at the TRIGA reactor in the Texas A\&M Nuclear Science Center. Three of the resulting sources, denoted $C 1, O 1$, and $O 2$ and described in Table I, were selected for measurement. As seen in Table I, for all three sources the difference in the calculated self-absorption between the $\mathrm{x}$ and $\gamma$ rays - the quantity that actually affects our results-is a small fraction of a percent. We made the correction nonetheless.

\section{B. Radioactive decay measurements}

Of the seven naturally occurring osmium isotopes present in our irradiated samples, only three could lead to radioactive products that might interfere with the activity we wish to study. The most abundant one, ${ }^{192} \mathrm{Os}(41.0 \%)$, produces $30.1-\mathrm{h}{ }^{193} \mathrm{Os}$, which $\beta^{-}$decays to stable ${ }^{193}$ Ir with the emission of iridium $\mathrm{x}$ rays and numerous $\gamma$ rays. To avoid contamination from this decay, we waited two weeks after source preparationmore than ten ${ }^{193}$ Os half-lives-before recording spectra for analysis. Although this decay also populates the 10.5-d metastable state of ${ }^{193} \mathrm{Ir}$, it is by such a weak branch that the 


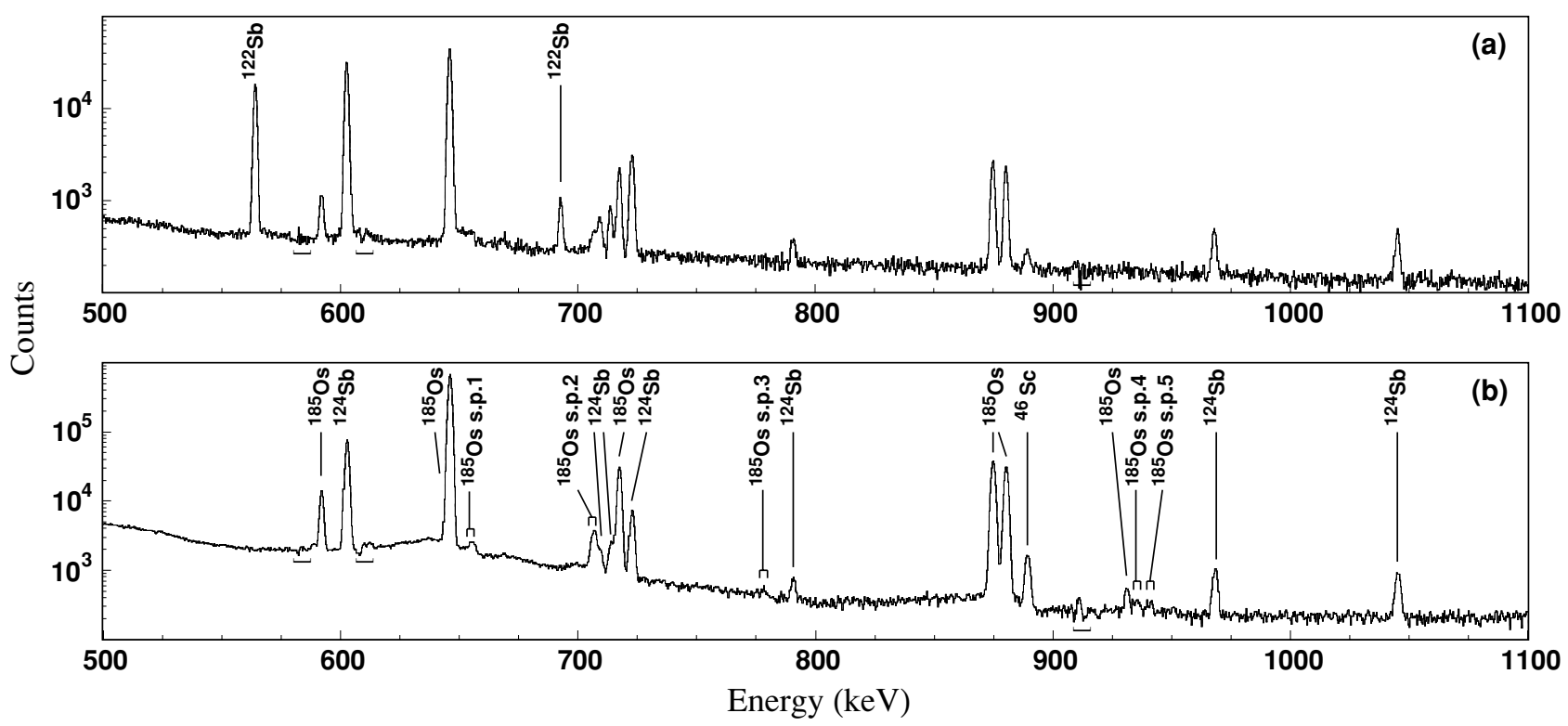

FIG. 2. A portion of the $C 1$ spectrum from the first series of measurements [part (a)] and of the $O 2$ spectrum from the third series (b) for the energy interval 500-1100 keV. Peaks are identified by their $\beta$-decay parent, with only short-lived parents being marked in the top panel, and longer-lived ones in the lower panel. Observed $\gamma$-x-ray coincidence sum peaks (s.p.) from ${ }^{185}$ Os decay are also marked: s.p. 1 designates the sum with rhenium $L \mathrm{x}$ rays, and s.p.2-5 that with rhenium $K$ x rays. The horizontal brackets located near the energy axis indicate regions where peaks in the subtracted background occur, and anomalous fluctuations can thus be expected.

$\mathrm{x}$ rays from that state's decay are negligible in our measurement. Delayed counting also removed any activity from decay of the 13.1-h metastable state in ${ }^{191}$ Os, which was produced, along with our activity of interest, from neutron capture on ${ }^{190} \mathrm{Os}$, the second most abundant isotope $(26.4 \%)$.

Finally, 93.6-d ${ }^{185} \mathrm{Os}$ is produced from the least abundant isotope, ${ }^{184} \mathrm{Os}(0.02 \%)$. Its small natural abundance is counterbalanced by a huge neutron-activation cross section (3000 b). However, it decays by electron capture to ${ }^{185} \mathrm{Re}$, and the rhenium $\mathrm{x}$ rays are well resolved from those of iridium.

We acquired spectra from all three sources using the same procedure as described previously for the ${ }^{193} \mathrm{Ir}^{m}$ experiment [1]. In particular, we used our HPGe detector, which has been efficiency calibrated to $0.15 \%$ relative precision between $50 \mathrm{keV}$ and $1.4 \mathrm{MeV}$ [12-14] at a source-detector distance of $151 \mathrm{~mm}$. Three series of spectra were recorded: the first, consisting of spectrum 1 with source $C 1$, and spectrum 2 with source $O 1$, began 15 days (one half-life of ${ }^{191} \mathrm{Os}$ ) after activation; the second, consisting of spectra 3-5 with $C 1, O 1$, and $O 2$, respectively, began about 1.5 half-lives later; and the third, consisting of spectra 6 and 7 with $C 1$ and $O 2$, respectively, began about 2.5 half-lives after that. Separate background spectra were recorded for each series. Each of the seven recorded spectra contained 4-10 million events in the $K$ $\mathrm{X}$ ray peaks.

\section{ANALYSIS}

Our analysis used the same methodology as described previously [1]. We first identified impurities, then made appropriate corrections to account for their effects. We also made the adjustments required to account for the Lorentzian shape of the X-ray peaks.

\section{A. Impurity identification}

All recorded spectra were carefully searched for impurities with rates above a few hundredths of a percent of the iridium $K \mathrm{x}$ rays, and their sources were identified by relative $\gamma$-ray intensities and decay curves. The results are illustrated in Fig. 2, where the region between 500 and $1100 \mathrm{keV}$ is shown from a spectrum in the first series and from another in the third series. Peaks are identified in these spectra, and impurity data are summarized in Table II. All peaks in all seven recorded spectra were identified and assigned to one of the $\beta$-decay parents listed in Table II.

It can be seen in Table II that, apart from trace amounts remaining from ${ }^{193} \mathrm{Os}$, only one observed isotope, ${ }^{122} \mathrm{Sb}$, had a half-life shorter than that of ${ }^{191} \mathrm{Os}$, and in all cases, impurity activities relative to that of ${ }^{191}$ Os are reasonably low. Note that the impurities listed in the top section of Table II are common to all sources; the ${ }^{181} \mathrm{Hf}$, listed in the last row, was only found in the spectra of the oxide sources.

\section{B. Peak ratios}

The spectrum in Fig. 3 shows the energy region of interest for this measurement, where the $K$ x rays and the $129.4-\mathrm{keV}$ $\gamma$ ray from ${ }^{191} \mathrm{Ir}$ appear. As in Ref. [1], we determined all peak areas with GF2, the least-squares peak-fitting program in the RADWARE series [15]. Because of the presence of rhenium $K \mathrm{x}$ rays, the whole group of $\mathrm{x}$ rays between $58 \mathrm{keV}$ and $80 \mathrm{keV}$ 
TABLE II. Identified impurities and their properties listed from higher to lower mass numbers. The absolute activities in column 3 and the relative activities in column 4 both refer to the time at which the $C 1$ source spectrum of the first series was recorded. Since ${ }^{181} \mathrm{Hf}$ was found only in spectra recorded for osmium oxide sources, it is listed separately in the last row, where the data refer to the third-series $O 2$ spectrum. All other impurities are common to both chloride and oxide sources.

\begin{tabular}{lccc}
\hline \hline $\begin{array}{l}\text { Parent } \\
\text { nucleus }\end{array}$ & $T_{1 / 2}$ & $\begin{array}{c}\text { Absolute } \\
\text { activity } \\
(\mathrm{Bq})\end{array}$ & $\begin{array}{c}\text { Activity } \\
\text { relative to } \\
191\end{array}$ \\
\hline${ }^{193} \mathrm{Os}(\%)$ \\
${ }^{192} \mathrm{Ir}$ & $1.255 \mathrm{~d}$ & 92 & 0.09 \\
${ }^{185} \mathrm{Os}$ & $73.8 \mathrm{~d}$ & 49 & 0.05 \\
${ }^{141} \mathrm{Ce}$ & $93.6 \mathrm{~d}$ & 7163 & 6.99 \\
${ }^{122} \mathrm{Sb}$ & $32.5 \mathrm{~d}$ & 103 & 0.10 \\
${ }^{124} \mathrm{Sb}$ & $2.72 \mathrm{~d}$ & 2768 & 2.70 \\
${ }^{65} \mathrm{Zn}$ & $60.2 \mathrm{~d}$ & 3981 & 3.89 \\
${ }^{60} \mathrm{Co}$ & $244 \mathrm{~d}$ & 19 & 0.02 \\
${ }^{46} \mathrm{Sc}$ & $5.27 \mathrm{y}$ & 4 & 0.01 \\
& $83.8 \mathrm{~d}$ & 21 & 0.02 \\
${ }_{\mathrm{Summed}}$ & & 14201 & 13.9 \\
${ }^{191} \mathrm{Os}$ & $15.4 \mathrm{~d}$ & 102412 & \\
${ }^{181} \mathrm{Hf}{ }^{\mathrm{a}}$ & & 96 & 0.02 \\
\hline \hline
\end{tabular}

${ }^{\mathrm{a}}$ Only in oxide sources.

was fitted in a single operation. In analyzing the result, we also took into account the $K_{\alpha}-L$ x-ray coincident summing and corrected for the effects of Ge $\mathrm{x}$-ray escapes from the detector. The resultant areas, divided by detector efficiency and live time, are given as decay rates in Table III for the $K$ x rays (upper part) and for the 129.4-keV $\gamma$ ray (middle part). Table III also lists the corrections we applied for impurities in the $K$ x-ray peaks; no impurities were found to affect the $\gamma$ ray. Our calculations found that $\gamma$-ray summing corrections had the same effect on the $\mathrm{x}$ rays as on the $\gamma$ ray, and would therefore cancel in the ratio. As a result, we made no further corrections for summing.

The corrections for impurities that affect the $K \mathrm{x}$-ray peaks are listed immediately below the raw decay rate for the $\mathrm{x}$ rays. The first is due to the $K \mathrm{x}$ rays resulting from internal conversion of the weak 82.4-keV transition in ${ }^{191} \mathrm{Ir}$ - see Fig. 1. The corresponding $\gamma$-ray peak is visible yet small in all spectra, but it is contaminated with $K_{\beta}-L$ coincident $\mathrm{x}$ rays from the converted $129.4-\mathrm{keV}$ and $41.8-\mathrm{keV}$ transitions. Consequently, in correcting for its effect, we used its known decay rate relative to the 129.4-keV transition [11].

The next listed impurity arises from the $\beta$ decay of ${ }^{192} \operatorname{Ir}$ to both platinum and osmium isobars; the resulting $K$ x rays are very close in energy to those of iridium and were not resolved. Their relative contribution was about a factor of two greater in spectra obtained from the oxide sources than in those obtained from the chloride source, and grew in time.

The remaining two impurities listed in Table III turn out to be negligible. The first-series measurements did show evidence of some remaining ${ }^{193}$ Os activity, but it had only a tiny effect on the x-ray peak. Similarly, the oxide sources showed evidence of

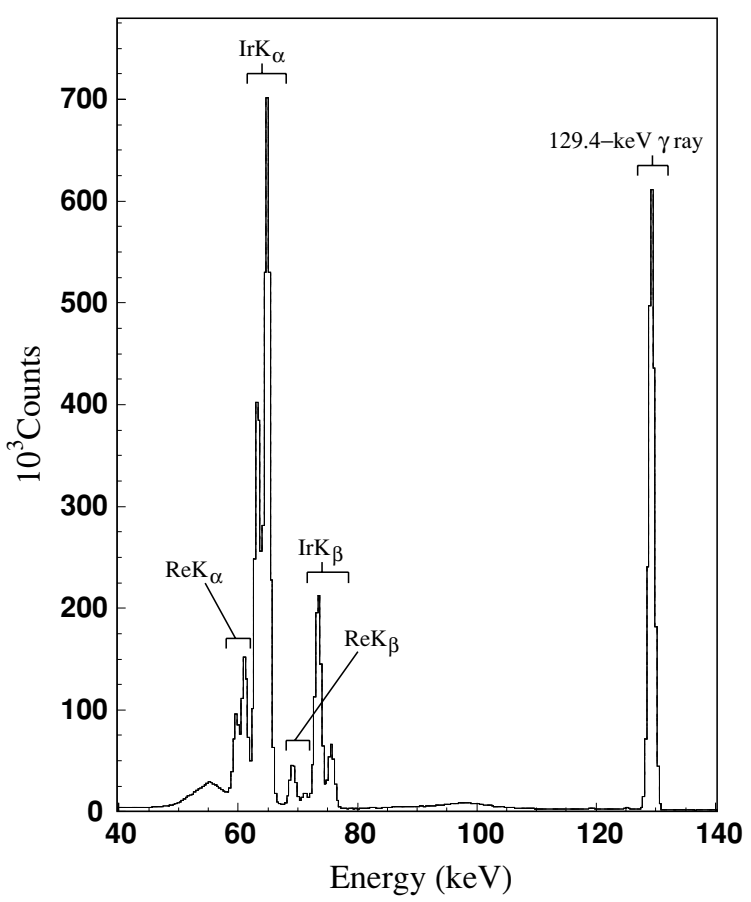

FIG. 3. Portion of the spectrum recorded from the $C 1$ source during the second series of measurements; it shows the $K \mathrm{x}$ rays and $129.4-\mathrm{keV} \gamma$ ray from the decay of the 129.4-keV excited state in ${ }^{191} \mathrm{Ir}$. Also marked are the $K \mathrm{x}$ rays of rhenium following ${ }^{185} \mathrm{Os}$ decay.

${ }^{181} \mathrm{Hf}$, which would have produced tantalum $K_{\beta}$ x rays within the $\mathrm{x}$-ray peaks of interest, but the amount was too weak to be of any concern.

The decays of the impurity-subtracted $K \mathrm{x}$ rays and the 129.4-keV $\gamma$ rays are shown in Fig. 4, where they are compared to decay curves calculated with the known half-life of ${ }^{191}$ Os. The agreement is excellent.

\section{Lorentzian correction}

We use the Gaussian function in GF2 to fit our experimental data in order to achieve the necessary consistency with our

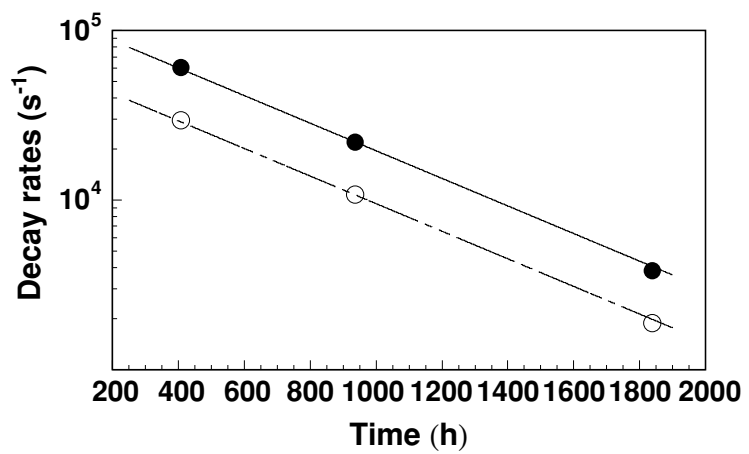

FIG. 4. Decay curves of the impurity-subtracted $K$ x rays (solid circles) and 129.4-keV $\gamma$ rays (open circles) in ${ }^{191} \mathrm{Ir}$. The lines are fitted decay curves with a fixed half-life of $15.5 \mathrm{~d}$ for the ${ }^{191} \mathrm{Os}$ parent activity. The data are from the osmium-chloride source spectra (numbers 1, 3, and 6 in Table III). 
TABLE III. Decay rates and impurity corrections for the $\left(K_{\alpha}+K_{\beta}\right) \times$ rays from ${ }^{191}$ Ir (upper section), and decay rates for the $129.4-\mathrm{keV}$ $\gamma$ ray (middle part). The lower section presents the activity ratios and their average. Spectra 1 and 2 were taken after about one half-life of ${ }^{191}$ Os; spectra 3-5, after an additional 1.5 half-lives; and spectra 6 and 7, after a further 2.5 half-lives. More specific details are given in Sec. II B of the text.

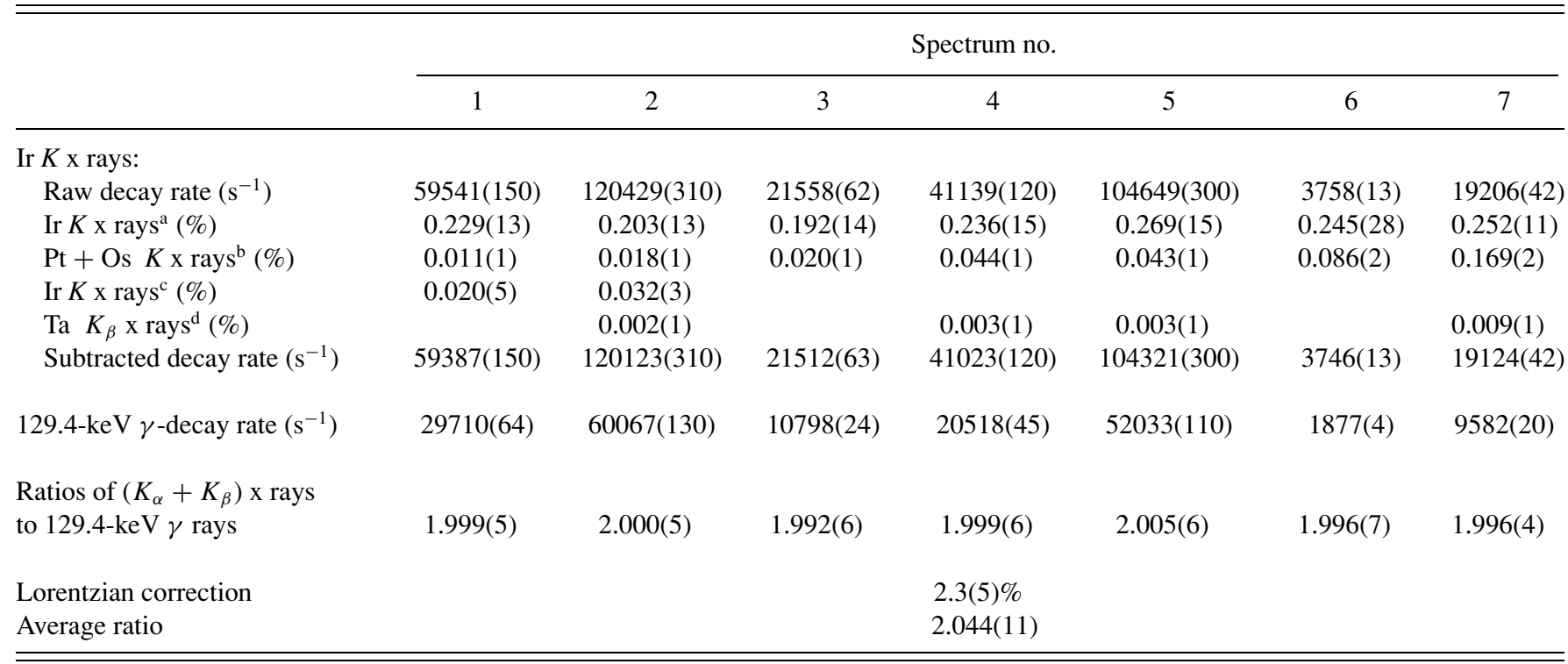

${ }^{\mathrm{a}}$ From internal conversion of $82.4-\mathrm{keV} \gamma$ ray of ${ }^{191}$ Os.

${ }^{\mathrm{b}}$ From ${ }^{192}$ Ir decay.

${ }^{c}$ From ${ }^{193}$ Os decay; only in spectra acquired first.

${ }^{\mathrm{d}}$ From ${ }^{181} \mathrm{Hf}$ decay; only in spectra of $\mathrm{OsO}_{2}$ sources.

efficiency-calibration procedures. However, as explained in our previous paper [1], this approach alone does not do justice to x-ray peaks, whose shapes reflect the finite widths of the atomic levels responsible for them. As before, we computed a simulated spectrum using realistic Voigt-function shapes for the X-ray peaks. Because we fitted the measured x-ray peaks as a group, we generated the whole group with the tabulated relative $x$-ray intensities and then scaled them to the number of counts actually obtained in our experiment. The simulated spectrum was then analyzed with GF2 following exactly the same fitting procedure as was used for the real data. We could then compare the areas obtained by GF2 for the iridium X-ray peaks with the actual peak areas used in the simulation. As shown in Table III, we found that $2.3(5) \%$ of x-ray peak areas was lost in the Gaussian fit.

\section{RESULTS AND DISCUSSION}

The ratio of $\mathrm{x}$ rays to $129.4-\mathrm{keV} \gamma$ rays is listed in Table III for each spectrum, and the average ratio, corrected for the Lorentzian effect, appears at the bottom. Note that the uncertainty on each ratio properly accounts for detector efficiency, incorporating the relative-efficiency uncertainty for each spectrum ratio, and including it only after averaging in the final result. Actually, the Lorentzian correction is the largest one we apply to our result, and its uncertainty is the biggest single contributor to the final uncertainty.

\section{A. Mixing ratio for the 129.4-keV transition}

Before proceeding to extract any information from our measured ratio, we must deal with the important fact that the $129.4-\mathrm{keV}$ transition in ${ }^{191}$ Ir has mixed $M 1+E 2$ multipolarity. Since the $B(M 1)$ for this transition is known to be 0.0185 Weisskopf units [11], which is well within the range of nonhindered transitions, penetration effects are small, and the mixing ratio enters directly into the calculated conversion coefficient according to the relation

$$
\alpha_{K}=\frac{\alpha_{K}^{M 1}+\delta^{2} \alpha_{K}^{E 2}}{1+\delta^{2}},
$$

where $\delta$ is the mixing ratio. There are only two reasonably precise measurements of $\delta$ in the literature that are themselves independent of calculated conversion coefficients: $-0.398(20)$ [16] and $-0.402(7)$ [17]. Both were based on the measured angular correlation of $129.4-\mathrm{keV} \gamma$ rays. The weighted average of these two results is also $-0.402(7)$, and it is this value, together with Eq. (2), that we use in the following sections in calculating $\alpha_{K}$ for the $129.4-\mathrm{keV}$ transition.

It is interesting to note that there are two other measurements of comparable precision for the magnitude of $\delta-0.395(15)[18]$ and $0.402(4)$ [19]—which were obtained from the measured ratios of $L_{1,2,3}$-shell conversion electrons. In the original papers, the mixing ratio was extracted by comparison with tabulated ICCs available at the time: those from Refs. [2] and [3], respectively. Since the $L$-shell conversion coefficients are rather insensitive to the treatment of the 
TABLE IV. Theoretical values of $\alpha_{K}$ for the 80.2-keV $M 4$ transition in ${ }^{193} \mathrm{Ir}$, and the $129.4-\mathrm{keV}(M 1+E 2)$ transition in ${ }^{191} \mathrm{Ir}$, together with their ratio $R$, based on different theoretical models for dealing with the $K$-shell vacancy. Shown also are the corresponding percentage deviations $\Delta$, from the experimental value $R(\operatorname{expt})=48.29(39)$. For a description of the various models used to determine the theoretical ratios, see Ref. [1].

\begin{tabular}{lrrrr}
\hline \hline Model & {$\left[\alpha_{K}\right]_{193}$ Ir } & {$\left[\alpha_{K}\right]_{191}$ Ir } & $R$ & $\Delta(\%)$ \\
\hline No hole & & & & \\
Hole, frozen orbitals & $103.3(3)$ & $2.147(8)$ & $48.1(2)$ & $0.3(9)$ \\
Hole, SCF of ion & $99.7(3)$ & $2.147(8)$ & $46.4(2)$ & $3.9(9)$ \\
\hline \hline
\end{tabular}

atomic hole in their calculation, we have now used the recent tables of Band et al. [6] to reanalyze the measured electron ratios in Refs. [18,19]. The reanalyzed results are $0.404(15)$ and $0.394(4)$, respectively. Both are consistent with the value we obtained from the ICC-independent measurements, particularly when one takes into account that the authors responsible for the latter, remarkably precise result [19] admit several times in their paper that "the experimental error bars may have occasionally been underestimated."

\section{B. Comparison of ICCs in ${ }^{191} \mathrm{Ir}$ and ${ }^{193} \mathrm{Ir}$}

Both in this measurement and in our previous one of the 80.2-keV transition from ${ }^{193} \mathrm{Ir}^{m}$ [1], we measured the product $\alpha_{K} \omega_{K}$. Since the iridium fluorescence yield is common to both, the ratio of these two results becomes independent of $\omega_{K}$. Thus,

$$
R=\frac{\left[\alpha_{K} \omega_{K}\right]^{193} \mathrm{Ir}}{\left[\alpha_{K} \omega_{K}\right]_{191} \mathrm{Ir}}=\frac{\left[\alpha_{K}\right]^{193} \mathrm{Ir}}{\left[\alpha_{K}\right]_{191} \mathrm{Ir}}=\frac{98.7(6)}{2.044(11)}=48.29(39) .
$$

This result can now be compared with the calculated ratios, obtained with different hole treatments, shown in Table IV. The calculated ICCs for the ${ }^{193} \mathrm{Ir}$ transition were taken from our previous paper [1]; those for ${ }^{191} \mathrm{Ir}$ were calculated with $129.415(13) \mathrm{keV}$ used for the transition energy [11]. The fifth column in Table IV gives the percentage difference between experiment and theory, $\Delta$, where

$$
\Delta=\frac{R(\text { expt })-R(\text { theor })}{R(\text { expt })} \times 100 \% .
$$

Clearly, the best agreement is obtained for the calculations using the so-called frozen orbitals approach. This is the same conclusion we reached in Ref. [1], but here the conclusion is completely independent of the fluorescent yield, which was not the case before.

\section{Determination of fluorescence yield $\omega_{K}$}

It can be seen from Table IV that the calculated value of $\alpha_{K}$ for the $129.4-\mathrm{keV}$ transition in ${ }^{191} \mathrm{Ir}$, which we have studied in this work, is very nearly independent of the model used to deal with the hole in the atomic $K$ shell. We, of course, chose the transition for this very reason, so that we could extract an experimental value for $\omega_{K}$ from the measured product $\alpha_{K} \omega_{K}$. Taking a "model-independent" theoretical value from the table of $\alpha_{K}=2.142(11)$, and our experimental result of $\alpha_{K} \omega_{K}=$ 2.044(11) from Table III, we obtain the result $\omega_{K}=0.954(9)$.

Our measured result compares very favorably with the predicted value $\omega_{K}=0.958$ (4) obtained [9] from the interpolation of a semi-empirical fit to experimental data. The interpolation was required because no experimental values previously existed at all between $Z=73$ and $Z=80$. We can now confirm the interpolation and, arguably, support the very small $0.4 \%$ uncertainty assigned in Ref. [9] to the $\omega_{K}$ values derived from the semi-empirical fit. Since we used the interpolated value in our ${ }^{193} \operatorname{Ir}^{m}$ measurement [1], we can again confirm the conclusions drawn in that reference.

\section{CONCLUSIONS}

We undertook the present study of ${ }^{191}$ Ir to improve and refine the test of ICC calculations that we began with a recent measurement [1] on ${ }^{193} \mathrm{Ir}^{m}$. The measurement of the product $\alpha_{K} \omega_{K}$ reported here allows us to take two alternative approaches to the ICC issue. In the first, we compare the ratio of the ${ }^{191} \mathrm{Ir}$ and ${ }^{193} \mathrm{Ir}^{m}$ measurements to produce a test that is independent of the iridium fluorescence yield. In the second, we make use of the relative independence of the ${ }^{191} \operatorname{Ir} \alpha_{K}$ value to the theoretical model used to deal with the $K$-shell hole, and extract a value for the iridium fluorescence yield, which we can compare to the value used in the earlier test [1]. In both cases, our result strongly confirms our earlier conclusion: that the electron hole must be accounted for in the calculation of internal-conversion coefficients, and that the "frozen orbital" approximation shows the best agreement with experiment.

We still plan to measure ICCs for other transitions that are sensitive to the atomic vacancy in order to ensure that our conclusions are universally applicable.

\section{ACKNOWLEDGMENTS}

The work of the Texas A\&M authors is supported by the U.S. Department of Energy under Grant No. DE-FG0393ER40773, and by the Robert A. Welch Foundation. The work of M. T. is supported by the Russian Foundation for Basic Research under Grant No. 02-02-17117, and by U. S. Defense Threat Reduction Agency Contract No. DTRA01 01-P-0134.
[1] N. Nica, J. C. Hardy, V. E. Iacob, S. Raman, C. W. Nestor Jr., and M. B. Trzhaskovskaya, Phys. Rev. C 70, 054305 (2004).

[2] L. A. Sliv and I. M. Band, Tables of Gamma-Ray Internal Conversion Coefficients, Part I: K Shell (Academy of Sciences USSR, Leningrad, 1956); Part II: L Shell (Academy of Sciences USSR, Leningrad, 1958); see also Gamma Rays, edited by L. A. Sliv (Academy of Sciences USSR, Leningrad, 1961), p. 318 (in Russian); see also 
Alpha-, Beta-, and Gamma-Ray Spectroscopy, edited by K. Siegbahn (North-Holland, Amsterdam, 1965) p. 1639.

[3] R. S. Hager and E. C. Seltzer, Nucl. Data, Sec. A 4, 1 (1968).

[4] F. Rösel, H. M. Fries, K. Alder, and H. C. Pauli, At. Data Nucl. Data Tables 21, 91 (1978).

[5] I. M. Band and M. B. Trzhaskovskaya, Leningrad Nuclear Physics Institute Report, 1978 (in Russian, unpublished).

[6] I. M. Band, M. B. Trzhaskovskaya, C. W. Nestor, Jr., P. Tikkanen, and S. Raman, At. Data Nucl. Data Tables 81, 1 (2002).

[7] I. M. Band and M. B. Trzhaskovskaya, At. Data Nucl. Data Tables 55, 43 (1993).

[8] S. Raman, C. W. Nestor, Jr., A. Ichihara, and M. B. Trzhaskovskaya, Phys. Rev. C 66, 044312 (2002).

[9] E. Schönfeld and H. Janssen, Nucl. Instrum. Methods Phys. Res., Sect. A 369, 527 (1996).

[10] J. H. Hubbell, P. N. Trehan, N. Singh, B. Chand, D. Mehta, M. L. Garg, R. R. Garg, S. Singh, and S. Puri, J. Phys. Chem. Ref. Data 23, 339 (1994).
[11] Evaluated Nuclear Structure Data File, maintained by the National Nuclear Data Center, Brookhaven National Laboratory: http://www.nndc.bnl.gov; The Isotopes Project, Lawrence Berkeley National Laboratory: http://ie.lbl.gov./toi.

[12] J. C. Hardy, V. E. Iacob, M. Sanchez-Vega, R. T. Effinger, P. Lipnik, V. E. Mayes, D. K. Willis, and R. G. Helmer, Appl. Radiat. Isotopes 56, 65 (2002).

[13] R. G. Helmer, J. C. Hardy, V. E. Iacob, M. Sanchez-Vega, R. G. Neilson, and J. Nelson, Nucl. Instrum. Methods Phys. Res. A 511, 360 (2003).

[14] R. G. Helmer, N. Nica, J. C. Hardy, and V. E. Iacob, Appl. Radiat. Isotopes. 60, 173 (2004).

[15] D. Radford (private communication).

[16] A. V. Davydov, G. R. Kartashov, and Yu. V. Khrudev, Yad. Fiz. 7, 735 (1968); Sov. J. Nucl. Phys. 7, 447 (1968).

[17] E. Bezakova, A. E. Stuchbery, H. H. Bolotin, W. A. Seale, S. Kuyucak, and P. Van Isaker, Nucl. Phys. A669, 241 (2000).

[18] B. I. Deutch and P. Hornshoy, Nucl. Phys. 53, 497 (1964).

[19] D. S. Brenner and M. L. Perlman, Nucl. Phys. A181, 207 (1972). 